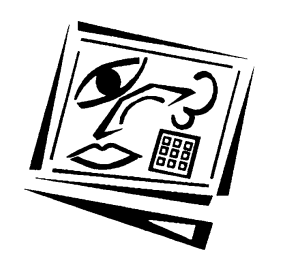

\title{
Presentation time concerning system-paced multimedia instructions and the superiority of learner pacing
}

\author{
Klaus D. Stiller, Kirstin Petzold and Peter Zinnbauer \\ University of Regensburg
}

\begin{abstract}
The superiority of learner-paced over system-paced instructions was demonstrated in multiple experiments. In these experiments, the system-paced presentations were highly speeded, causing cognitive overload, while the learner-paced instructions allowed adjustments of the presentational flow to the learner's needs by pacing facilities, mostly leading to higher learning success. A research gap exists in investigating how effects depend on the speed of system-paced presentations. Hence, in this follow-up experiment to Stiller, Freitag, Zinnbauer and Freitag (2009), 142 university students received four web-based instructions consisting of static pictures and on-screen texts about the structure of the human eye. Three instructions were system-paced with a low, medium, and high speed, and one condition was learnerpaced. Students rated their mental effort while learning and performed tests of retention, labelling pictures, verbalising structural knowledge, and transfer. With learner pacing, students reported lower mental effort and were better in labelling pictures and verbalising structural knowledge. Surprisingly, the medium systempaced instruction resulted in the worst performance, which partly questions common explanations of pacing effects.
\end{abstract}

\section{Introduction}

A low interactivity level of instructional systems is given by enabling learners to pace an instruction (Sims, 1997, 2000), which is proposed to improve learning (Betrancourt, 2005; van Merriënboer \& Kester, 2005; Mayer, 2005). In a recent publication summarising the theoretical and empirical knowledge in respect of instructional multimedia design, van Merriënboer and Kester (2005) suggested the self-pacing principle as a design advice. This strategy proposes allowing learners to control the pace over a multimedia instruction consisting of text and pictures, because it has a positive effect on elaborative learning and transfer (van Merriënboer \& Kester, 2005). This principle subsumes two further suggested design principles: The segmentation and interactivity principles. The segmentation principle recommends presenting narrated dynamic visualisations (i.e., animations and videos) in learner-paced segments rather than as a continuous unit (Mayer, 2005), and the interactivity principle proposes allowing learners to control the pace over a dynamic presentation (Betrancourt, 2005). Both strategies are suggested because learners learn more effectively in computer-based environments when learner pacing is allowed.

The empirical base of the self-pacing principle was given by multiple experiments comparing system-paced and learner-paced instructions. An instruction was systempaced when the system determined the duration of information presented. Learner pacing was realised by allowing learners to decide to start a part of the presentation 
after the preceding part has finished or to choose when a presentation should be paused and played using interactivity facilities (Hasler, Kersten \& Sweller, 2007; Mayer \& Chandler, 2001; Schwan \& Riempp, 2004).

The superiority of learner pacing was observed using various methods: Less mental effort while learning (Hasler et al., 2007; Moreno, 2007; Stiller et al., 2009), less time on acquiring a skill (Schwan \& Riempp, 2004), and higher scores on tasks of retention, labelling pictures, transfer, knowledge application and structural knowledge (Hasler et al., 2007; Lusk et al., 2008; Mayer \& Chandler, 2001; Moreno, 2007; Stiller et al., 2009; Stiller \& Zinnbauer, 2011; Tabbers, 2002). Empirical evidence supporting advantages of learner pacing has been found with short (less than $10 \mathrm{~min}$ ) and longer lasting (up to $30 \mathrm{~min}$ ) instructions (Hasler et al., 2007; Mayer \& Chandler, 2001; Schwan \& Riempp, 2004; Stiller et al., 2009, and Lusk et al., 2008; Moreno, 2007; Stiller \& Zinnbauer, 2011; Tabbers, 2002, respectively), using narrated dynamic visualisations, such as animations and videos (Hasler et al., 2007; Mayer \& Chandler, 2001; Moreno, 2007; Stiller \& Zinnbauer, 2011), dynamic visualisations without text (Schwan \& Riempp, 2004), narrated dynamic presentations comprising a mixture of pictures, diagrams and onscreen text (Lusk et al., 2008) or static visualisations with visual, but not auditory text (Tabbers, 2002; Stiller et al., 2009), and with adults and children (Lusk et al., 2008; Mayer \& Chandler, 2001; Moreno, 2007; Schwan \& Riempp, 2004; Stiller et al., 2009; Stiller \& Zinnbauer, 2011; Tabbers, 2002, and Hasler et al., 2007, respectively). The above studies lacked experimental manipulation in a naturalistic setting; they were mostly conducted in laboratories by individually testing participants with narrated dynamic visualisations (Betrancourt, 2005; Mayer, 2005). Only Tabbers (2002) and Stiller et al. $(2009,2011)$ used online or classroom settings.

Older research about pacing in computer programs showed inconsistent results (Niemiec, Sikorski \& Walberg, 1996; Reeves, 1993; Williams, 1996) and thus is not helpful. Two problem areas were identified: A lack of theoretical foundations of the experiments and methodological problems (Lepper, 1985; Reeves, 1993; Williams, 1996). Especially the latter gave rise to essential criticism. Reeves (1993) and Williams (1996) blamed unclear, ambiguous definitions of the implemented types of learner control that render interpretations of results difficult, as well as using inadequate designs, which include poor instrumentation, control of procedures and sampling as well as means of data analysing. As a typical problem, Williams (1996) pointed at the confounding of control type (learner vs. system) with the amount of instruction. Often learners having control over an instruction incompletely study it, while the learners guided by the system simply receive more instruction. This problem frequently leads to a superiority of system-controlled instructions due to providing a more complete lesson.

\section{Explaining pacing effects}

Learners must accomplish all the processing necessary for learning within the capacity limits of working memory. Cognitive load theory (Sweller, 2005) especially emphasises the role of the working memory in learning and the problem of occupying its capacity by useful and unnecessary processes. Intrinsic, germane, and extraneous cognitive load are distinguished. Intrinsic load is created by task complexity, that is element interactivity, by the number of information elements that have to be processed simultaneously in working memory to be integrated into a new schema. Germane load is caused by processing information in order to build, automate, and store schemas. 
Extraneous load results from "inappropriate instructional designs that ignore working memory limits and fail to focus working memory resources on schema construction and automation" (Sweller, 2005, p. 26). The presentation manner of the material is often the main detrimental source of learning. When the three loads do not occupy the entire working memory capacity, some is still available for processing.

Learner pacing seems to be especially helpful when it can improve a presentational design in a way that greatly enhances information processing. This is true for dynamic presentations using speech and dynamic visualisations that bring learners to their limits by putting high extraneous load on working memory through a continuous and speedy presentation of information (Hasler et al., 2007; Mayer \& Chandler, 2001; Moreno, 2007). Such a presentation forces the learners to perceive and process information within a given, short time period. Learners only have limited time to extract relevant information and to maintain it in working memory until information is organised and integrated before the next presentational part has to be processed. The means of pacing used in the experiments brought two improvements to that kind of instructions: They enabled learners to adjust the information flow to their needs of information processing and segmented the continuous presentation into conceptually meaningful entities that might foster the construction of schemas. Thus, we have also at least two confounded factors in that kind of presentational manipulation.

The importance of information flow adjustment to the learners' speed of information processing was addressed by Mayer and Chandler (2001), who proposed that learner pacing reduces extraneous load on working memory and thus enables learners to build a more coherent mental model step by step. Learner pacing allows learners to stay longer on parts of information that are difficult to understand before the next part is processed (and thus results in an increase of study time). With additional time, more of the relevant information is processed into working memory as well as organised and integrated into a more coherent and complete mental model. This adjustment seems to be particularly helpful with time-dependent presentations, which only enable learners to perceive and process information within a given time period (Hasler et al., 2007; Mayer \& Chandler, 2001; Moreno, 2007; Schwan \& Riempp, 2004).

\section{Pacing and split-attention effects}

The explanation above of Mayer and Chandler (2001) is especially helpful with instructions that are likely to create split-attention effects. A split-attention effect might occur when learners are required to divide their attention between multiple sources of information that must be mentally integrated to achieve comprehension (Ayres \& Sweller, 2005). A central characteristic of the separated sources of information is that all sources are needed to reach full understanding. The sources can be separated in time or space. This means for spatial split-attention, for example, learners must search for corresponding information from concurrently presented visual texts and pictures, which often results in less relevant material being selected for further processing (especially with system-paced dynamic instructions), and then keep the information concurrently in working memory in order to enable integration processes. In respect of temporal split-attention, learners have to maintain relevant information from a piece of presentation in working memory until it can be integrated with relevant information from a subsequent part. If people have to split their attention between such sources of information, extraneous load of working memory will increase. If this increase overloads working memory, learning is hindered. Consequently, understanding and 
schema acquisition as well as schema automation are disturbed and reflected in performance losses (Ayres \& Sweller, 2005). Learner pacing might mean compensation (or only reduction) of split-attention effects. Compensation is reached by allowing learners to control the time spent on the task. Thus learners can have as much time as they need to select relevant information from disparate sources and to integrate them. This can mean that learners take the time in order to compensate spatial split-attention effects (Tabbers, 2002) or to accelerate studying a presentation in order to reduce the temporal separation of corresponding information (Stiller et al., 2009).

In this context of split attention, Tabbers (2002) and Stiller et al. (2009) have found an improvement in learning for visual texts with static visualisations by implementing a forward button to allow learner pacing. The visualisations did not change dramatically in time, that is, they were relatively stable and only parts of the visualisations were highlighted in correspondence to the presented text. A benefit of segmentation as with dynamic presentations was not given, because the material was already conceptually segmented. Additionally, Tabbers (2002) and Schmidt-Weigand (2006) investigated the effect of study time allowed by a presentational system to clarify effects of system pacing on split-attention effects and have found benefits of a prolonged study time. The studies are shortly reported in the following paragraphs.

Stiller et al. (2009) investigated the effects of pacing and text modality on learning in an online experiment. University students studied instructions on the human eye paced either by the system or the learner and using either visual or auditory texts with static pictures. Learner pacing reduced mental effort and promoted performance for instructions using visual texts. Surprisingly, the learner-paced, visual text instruction was studied faster than the system-paced version. This led to the interpretation that learners could easily compensate visual split-attention between on-screen texts and pictures and extract relevant information within the system-paced presentation periods per information unit, but were handicapped by waiting time for the next piece of information that interrupted processing. In other words, the effect of temporal splitattention was reduced by learner pacing.

Schmidt-Weigand (2006) investigated system pacing with animations on lightning formation. The animations were played in a slow, medium, and fast pace using either visual or auditory texts. A slower presentation rate led to marginally more success at retention and visual recall (drawing task), but not at transfer. Mental effort decreased significantly with expanded presentations for visual but not for auditory texts. Although Schmidt-Weigand (2006) used the most demanding presentation format of animation, he delivered only weak evidence for the assumption that prolonging presentation time allows an individual more adequate speed of information processing including a compensation of split-attention effect with visual text and pictures.

Tabbers (2002) used web-based lessons about a didactical model of instructional design (static diagrams were explained by auditory or visual texts), focusing on pacing and text modality. Information units were put in linear order, and when instructions were self-paced, learners could move forward along this line. In two experiments, the results showed that for on-screen texts mental effort was not influenced by pacing, performance of retention and transfer increased with more time on task when using system-paced instructions, and learner pacing additionally improved learning. Tabbers (2002) referred the improvement of learning with system-paced instructions to extended time on task, which is responsible for compensating split-attention effects, 
and argued that learner pacing additionally allows a higher quality of information processing with on-screen texts.

Moreover, Tabbers (2002) and Stiller et al. (2009) have shown that effects of learner pacing can depend on the text modality. They only found an improvement in learning for on-screen texts and static visualisations, whereas for auditory texts learner pacing was worthless or even detrimental in aspects of performance. The learner-paced, visual text instruction of Stiller et al. (2009) and Tabbers (2002) were also superior to the corresponding auditory text instructions.

Tabbers (2002) argued that learner pacing does not only allow control over the time spent on instructions and thus compensation of split-attention effects with visual texts, it additionally allows a higher quality of information processing with visual texts. He proposed that reading texts is a more active process that enables flexible as well as strategic processing of information. Jumping back and forth in written text to access or revisit pieces of information, for example, is much easier than with audio. A more flexible work-through might lead to a greater chance of retaining corresponding verbal and pictorial information in working memory, of improving mental representations by noticing formerly unnoticed but essential information, of mapping verbal and pictorial information adequately, and of using prior knowledge for knowledge construction. Perhaps learner pacing allows learners to transfer their successful and schematised reading behaviour to new instructional multimedia situations. For this reason, learnerpaced instructions with visual texts might better satisfy the learners' individual cognitive needs with regards to information processing. For the detrimental effects of pacing with auditory texts, Tabbers (2002) complementally proposed that listening is a rather passive process which makes it suitable for linear presentations with minimal interactivity, as when instructions are paced and sequenced by the system. Learner pacing is incompatible with normal listening behaviour.

\section{Research questions and hypotheses}

In the following experiment, the effects of instructional pacing on mental effort and performance were investigated. Therefore, students were asked to work on various performance tasks and to rate their mental effort while learning after studying an instruction composed of on-screen text and static pictures.

Firstly, the effect of pacing found by Stiller et al. (2009) with on-screen texts and pictures in an online setting should be replicated to gain supporting evidence of pacing effects with visual texts in more ecologically valid settings. Learners pacing the instruction should gain more knowledge and experience less mental effort than learners studying system-paced presentations (Stiller et al., 2009). The pace of a presentation determines the time available for information processing. If learners can affect the pace, they may choose one that matches their individual speed of comprehension. This also means that they can spend as much time as they need with the information presented, that is, learners are more likely to select relevant information and to organise and to integrate it adequately. Hence, we proposed that learner pacing is suitable for preventing working memory from being overloaded or at least for relieving working memory from extraneous load (Tabbers, 2002; SchmidtWeigand, 2006).

Secondly, the extent that presentational speeds of pacing by the system are reflected in learning and how this can be explained should be clarified. Learners studying a fast or 
slow system-paced presentation should have less success in the performance tasks and experience more mental effort than learners who learn with a medium system-paced instruction. A main cause of effects is seen in split-attention effects. Depending on the speed of presentation, a visual or temporal split-attention effect is seen as the main contributor to an assumed pacing effect. Therefore, fast and slow pacing should mainly lead to visual and temporal split-attention effects, respectively, and a medium presentation speed should minimise but not eliminate visual and temporal splitattention effects. Henceforward, results can be used to improve interpretation of modality effects (i.e., learners learn more effectively in computer and book based environments when spoken instead of visual texts are used; Ginns, 2005), when they help to clarify the mechanisms that underlie the processing of system-controlled presentations of on-screen text and pictures.

\section{Method}

\section{Participants}

Voluntarily, 219 students started an experimental session, but 77 data sets were not accepted due to the following aspects.

- Three students had participated in the experiment of Stiller et al. (2009).

- Two students did not speak German as their first language.

- 18 students showed irregularities in the participation.

- Seven students reloaded an instruction or were lurking before participating.

- Five students broke off after the prior knowledge test and before assignment to a treatment group.

- One student waited more than an hour after studying the presentation to rate loads and to work on the performance test.

- Two students of the learner-paced group studied for 12 and 47 seconds, which was deemed too short to learn seriously.

- Three students chose the option "unserious participant" in the pre-questioning.

- 54 students were eliminated due to two types of unknown causes titled, break-offs and missing data. Cases of break-offs and missing data were equally distributed over treatment groups (see Table 1).

- 27 break-offs of the instructions occurred.

- 27 data of load ratings and performance were missed, although instructions were studied.

Finally, 142 students from German universities contributed to the sample, with a mean age of 23.5 years $(S D=2.9)$ and a mean semester of study of $5.2(S D=2.8)$. Table 1 provides an overview of the frequencies of gender and university membership, descriptive statistics of age and semester of study, and the results of the tests concerning the comparability of treatment groups. Most students studied psychology or pedagogical subjects.

\section{Multimedia instructions}

The learning material covered the structure of the human eye. Therefore, a horizontal section through the eye was presented and the parts of the eye were explained by onscreen texts comprising 812 words (see Figure 1). Four levels of instructions were created. One instruction was learner-paced and three were system-paced with a low, 
medium, and high presentational speed. Learner pacing meant that the learner chose when the next text unit should be presented. System pacing meant that the instructional units were presented one after the other without a break in between. First, the picture was verbally introduced. Then 12 parts of the eye were explained in the following order: cornea, pupil, iris, lens, ciliary muscle, vitreous body, sclera, choroid, retina, optic disc, fovea, and optic nerve. While the text belonging to a pictorial part was read, this part was highlighted in green until the next text unit was presented.

Table 1: Break-offs, missing data, gender, university membership, descriptive statistics and tests concerning the comparability of treatment groups

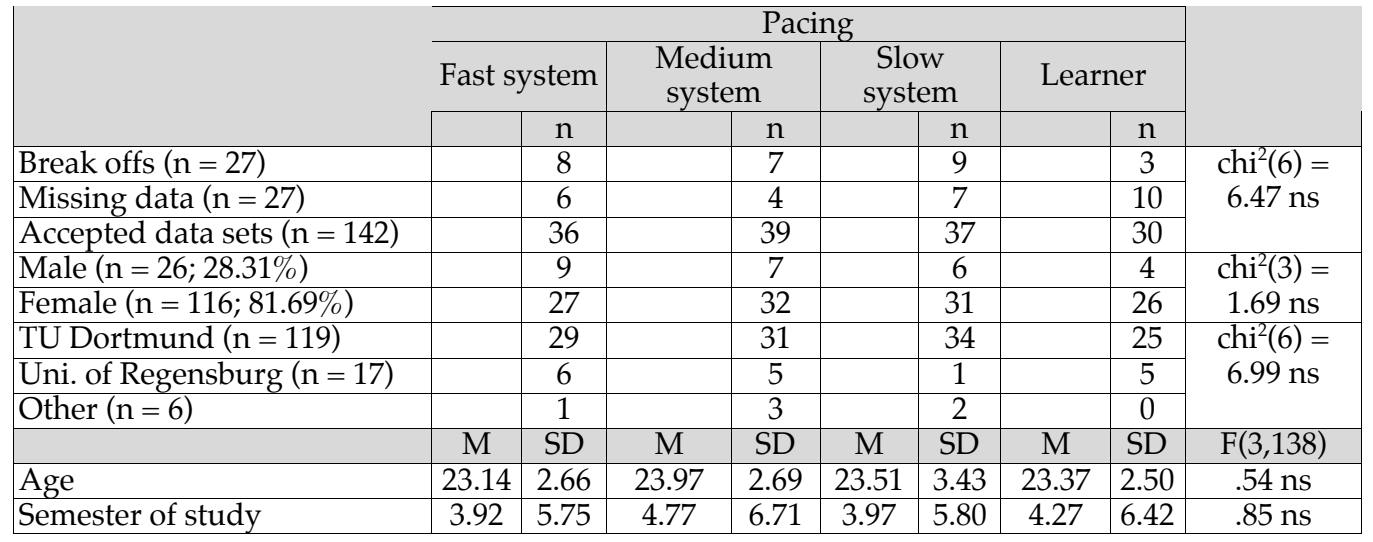

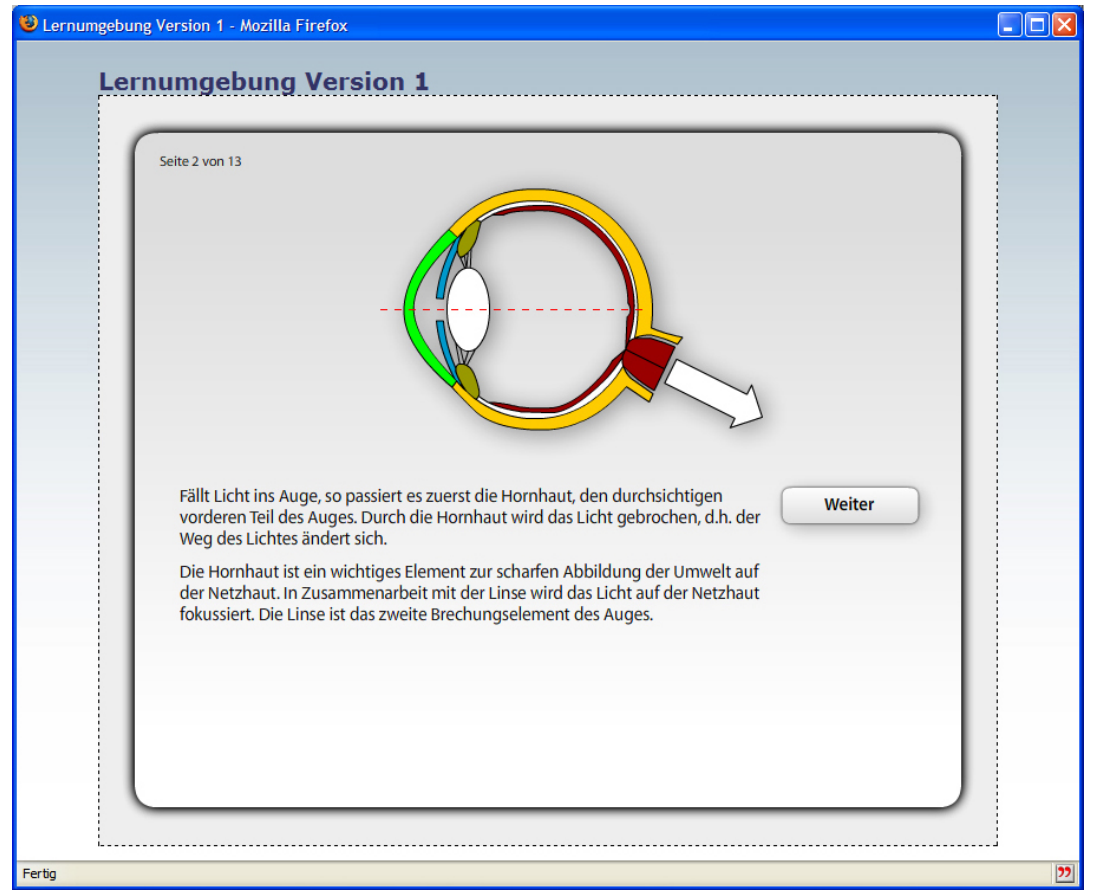

Figure 1: Screenshot of the learner-paced instruction. 


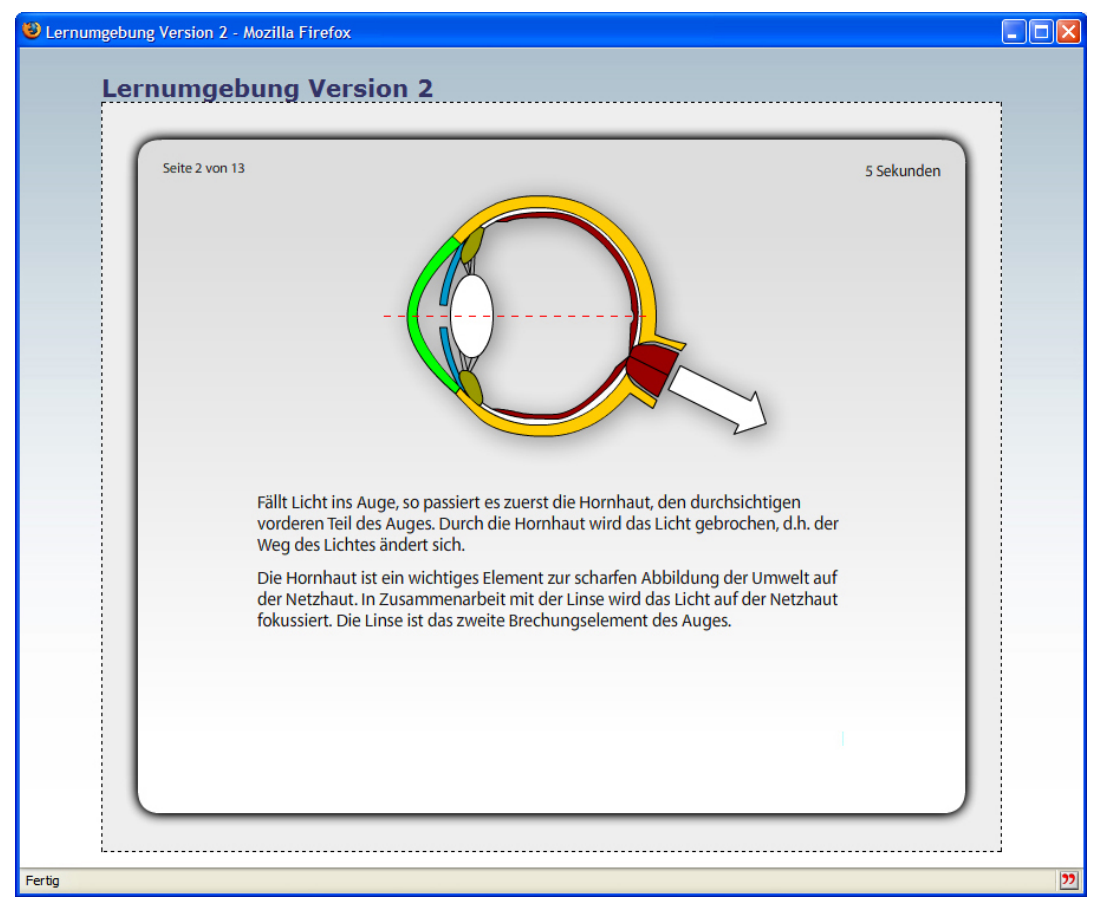

Figure 2: Screenshot of a system-paced instruction.

How were the presentation speeds of the system-paced instructions determined? Variances appeared due to the transfer rate of the web server. The low speeded presentation lasted 7:07 min on average $(=427$ seconds, $\mathrm{SD}=2.68)$ and corresponds to the system-paced, visual text instruction used by Stiller et al. (2009). The medium speeded presentation lasted 5:46 min on average $(=346$ seconds, $\mathrm{SD}=1.68)$ and was adjusted to the average study time of the learner-paced, visual text instruction used by Stiller et al. (2009). The average study time of the learner-paced instruction in this experiment was 5:31 $\mathrm{min}(=331$ seconds, $\mathrm{SD}=116.70)$. The high speeded presentation lasted 4:14 min on average $(=254$ seconds, $\mathrm{SD}=1.78)$ and was determined by subtracting the difference of the mean presentation time of the system-paced visual text instruction and the mean study time of the learner-paced visual text instruction from the mean study time of the learner-paced visual text instruction used by Stiller et al. (2009).

A couple of numbers that were placed in the top left-hand corner of the display signalled the number of text units in the sequence and at which position of the sequence the learner was. In addition, in the system-paced conditions, the presentation durations of the text units were shown via a count-down from total playing time to zero.

\section{Means of measurement}

Prior factual knowledge was measured by a recognition task comprising eight multiple choice questions: "What is regulated by the ciliary muscles?", "What happens in the optic chiasm?", "What is the function of the choroids?", "What kinds of photo 
receptors exist?", "What is the area of the retina called on which the projection of an object falls when it is fixated?", "What is the area of the brain called, which is mainly responsible for vision?", "What is the blind spot of an eye?", and "What cells of the retina build up the optic nerve?". Four answers were offered for each question along with an option to mark "I do not know". One of the answers was correct. Each answer was scored with a maximum of one point.

The presentations were followed by three questions assessing three types of cognitive load (Schmidt-Weigand, 2006). Overall load was subjectively rated by "How easy or difficult was it for you to learn something about the eye from the presentation you just saw?", intrinsic load by "How easy or difficult would you consider the content?", and extraneous load by "How pleasant or bothersome would you consider the presentation format?". Participants were instructed to place a check mark for each question on a 7point rating scale from very easy (pleasant), across easy (pleasant), rather easy (pleasant), medium, rather difficult (bothersome), difficult (bothersome), to very difficult (bothersome).

The performance test consisted of a verbal structure task, a transfer task, a pictorial labelling task, and a verbal retention task. Stiller et al. (2009) additionally showed some figures depicting what the students saw on their computers when actually performing the various tasks of the performance test.

- The structure task required the students to name the structures of the eye passed by a visual stimulus on its way through the eye, and to arrange them according to the sequence of contact (cornea, pupil, lens, vitreous body, retina, optic disk, and optic nerve). For each element listed in the correct order, a maximum of one point was scored. The elements that were mentioned without being in the list of correct answers (e.g. choroid) were also counted (superfluous details).

- The transfer task asked the learners to use their knowledge to find explanations for the following situation: "Imagine that a friend of yours no longer sees very well with one eye. What possible reasons are there for this? Please list all your ideas about what parts of the eye are malfunctioning!". Based on the presented information, 16 reasons were accepted. They were coded into nine items as multiple causes that referred to one part of the eye (e.g., lens is clouded or inflexible) were put together.

- Then the participants had to name nine parts of the eye (cornea, iris, lens, ciliary muscles, vitreous body, sclera, choroid, retina, and optic nerve). The same picture was used as in the presentations and the relevant parts were marked. For each item, 1 point was scored in maximum.

- Retention was assessed by five open questions coded into six items, which were scored with a maximum of one point: "Which process takes place in the retina?", "What is the function of the choroid?", "Which parts of the eye are responsible for a sharp projection of the world on the retina?" (coded into two items), "What is the function of the iris?", and "What elements form the optic nerve?".

Table 2 lists the number of items, mean, standard deviation, reliability (Cronbach's alpha) and objectivity of the measurement scales. Performance scales were normed to the range of 0 to 100, thus enabling scores to be interpreted as percent correct answers; for superfluous details, only their frequency was counted. All tests are assessed as criteria tests. Content validity of all performance tests is given; the questions of the performance tests were constructed from the presented instructional content. A second rater scored 40 performance tests $(28 \%)$ randomly drawn from all regular data sets. 
The Pearson correlation of sum scores was used as a measure of objectivity. The range of the cognitive load ratings was 1 to 7 . The computer registered the time needed for presentation.

Table 2: Features of the measurement scales

$\left(\right.$ alpha $=$ Cronbach's alpha; $r_{\text {interrater }}=$ objectivity; $\left.\mathrm{n}=142\right)$

\begin{tabular}{|l|c|c|c|c|c|}
\hline & Items & $\mathrm{M}$ & $\mathrm{SD}$ & alpha & $\mathrm{r}_{\text {interrater }}$ \\
\hline Prior knowledge & 8 & 34.51 & 27.16 & .73 & -- \\
\hline Overall load & 1 & 3.58 & 1.44 & -- & -- \\
\hline Intrinsic load & 1 & 3.68 & 1.20 & -- & -- \\
\hline Extraneous load & 1 & 3.33 & 1.56 & -- & -- \\
\hline Verbal retention & 6 & 38.44 & 29.51 & .76 & .99 \\
\hline Verbal structure: correct details & 7 & 56.84 & 29.44 & .75 & .90 \\
\hline Verbal structure: superfluous details & -- & .54 & .91 & -- & .99 \\
\hline Labelling pictures & 9 & 60.92 & 29.99 & .82 & .99 \\
\hline Transfer & 9 & 17.69 & 13.46 & .61 & .94 \\
\hline
\end{tabular}

\section{Procedure}

The experiment was conducted at distance via the Internet. Students were asked to participate at any time or place of their choice but should arrange for an undisturbed environment. After requesting the URL, the students were introduced to the experiment, its procedure, and the technical affordances. Next, they were asked about their sex, age, university membership, semester of study, and subject of study, and they had to commit that their participation is based on seriousness and compliance. Finally, they completed a test of prior knowledge. After this pre-testing, participants were randomly assigned to one of the four treatment groups: 36, 39, and 37 students served in the fast, medium, and slow system-paced groups, respectively, and 30 in the learner-paced group. Before starting the instruction, the groups were briefly introduced to its handling. Then the students learned with their treatment instruction. Subsequently, the students rated the overall difficulty of the presentation and the content as well as the pleasantness of the kind of presentation. Finally, they took a performance test. The whole experiment took about 18.54 minutes on average (SD = 5.76).

\section{Results}

Prior knowledge, which is known to influence learning outcomes, was planned to be used as a covariate in a series of one-way between-subjects analyses of covariance, but slopes of the regression lines representing the relationship between the dependent variables and the covariate in the four groups could not be considered as equal (for overall load, retention, labelling, and transfer, ANCOVAs resulted in significant interaction effects with $\mathrm{F}(3,134)>2.70, \mathrm{p} \leq .05$; for intrinsic load, extraneous load, correct and superfluous details of the verbal structure task, no significant interactions were found with $.62<\mathrm{F}(3,134)<1.63, \mathrm{p}>.18)$. Hence, the treatment groups were tested for differences in prior knowledge. As no differences were found (see Table 4), a series of one-way between-subjects analyses of variance was conducted with pacing serving as between-subject factor. As dependent variables, cognitive load and performance were assessed. Assumptions of normality (see Table 3) and homogeneity of variance could mostly be retained. Normality of distributions was mainly violated concerning data of reporting superfluous details (right skewed distributions; many 
students did not report any superfluous details), homogeneity of variance was violated concerning data of correct details in the verbal structure task (Levene statistic $(3,138)=$ $3.43, \mathrm{p} \leq .05)$ and labelling pictures (Levene statistic $(3,138)=3.99, \mathrm{p} \leq .01$ ). As an ANOVA is regarded to be very robust to most plausible violations of the usual assumptions (Bortz, 2005), the strategy of analyses was not changed. Controlling effects via the robust Brown-Forsythe-Method confirmed results. Partial eta-squared effect sizes were computed.

Table 3: Skewness (Sk) and Kurtosis (K) of the distributions of the dependent variables and results of the Kolmogorov-Smirnov tests $\left({ }^{*} \mathrm{p} \leq .05 ;{ }^{* *} \mathrm{p} \leq .01\right)$

\begin{tabular}{|c|c|c|c|c|c|c|c|c|c|c|c|c|}
\hline & \multicolumn{12}{|c|}{ Pacing } \\
\hline & \multicolumn{3}{|c|}{ Fast system } & \multicolumn{3}{|c|}{ Medium system } & \multicolumn{3}{|c|}{ Slow system } & \multicolumn{3}{|c|}{ Learner } \\
\hline & Sk & K & $\mathrm{Z}$ & Sk & $\mathrm{K}$ & $\mathrm{Z}$ & Sk & $\mathrm{K}$ & $\mathrm{Z}$ & Sk & $\mathrm{K}$ & $\mathrm{Z}$ \\
\hline Overall load & -.53 & -.13 & 1.12 & .25 & -.61 & 1.18 & .49 & -.75 & 1.30 & .56 & .07 & .99 \\
\hline Intrinsic load & .32 & -.93 & 1.23 & -.16 & -.89 & 1.19 & -.43 & -.95 & 1.30 & .59 & -.61 & $1.63^{* *}$ \\
\hline Extraneous load & .10 & -.61 & .97 & .37 & -.91 & 1.10 & .49 & .19 & .90 & .48 & -.78 & 1.16 \\
\hline Verbal retention & .18 & -1.01 & .73 & .49 & -1.18 & 1.22 & .46 & -.84 & .77 & -.08 & -.78 & .45 \\
\hline $\begin{array}{l}\text { Verbal structure: } \\
\text { correct details }\end{array}$ & -.74 & -.63 & $1.49^{*}$ & -.27 & -1.34 & 1.22 & -.84 & .17 & 1.32 & -.54 & -.20 & 1.17 \\
\hline $\begin{array}{l}\text { Verbal structure: } \\
\text { superfluous details }\end{array}$ & 2.27 & 4.70 & $2.48^{\star *}$ & 1.64 & 1.69 & $2.57^{* *}$ & 1.50 & 2.65 & $1.91^{* *}$ & 2.40 & \begin{tabular}{|l|}
5.64 \\
\end{tabular} & $2.31^{* *}$ \\
\hline Labelling pictures & -.82 & -.42 & 1.12 & -.02 & -1.50 & .90 & -.10 & -.68 & .64 & -.96 & 20 & .89 \\
\hline Transfer & .30 & -.61 & .71 & .77 & .14 & 1.26 & .76 & .33 & 1.15 & .66 & -.07 & .91 \\
\hline
\end{tabular}

The ANOVAs revealed a significant effect of pacing on extraneous and overall load, but no effect on intrinsic load (see Table 4). As shown by contrasts (see Table 5), with learner pacing, students experienced lower extraneous load than with system pacing and a corresponding effect could be observed for overall load. The means of the system-paced groups did not differ significantly.

Table 4: Results of the ANOVAs and partial eta-squared effect sizes ( $\left.{ }^{* *} \mathrm{p} \leq .01\right)$

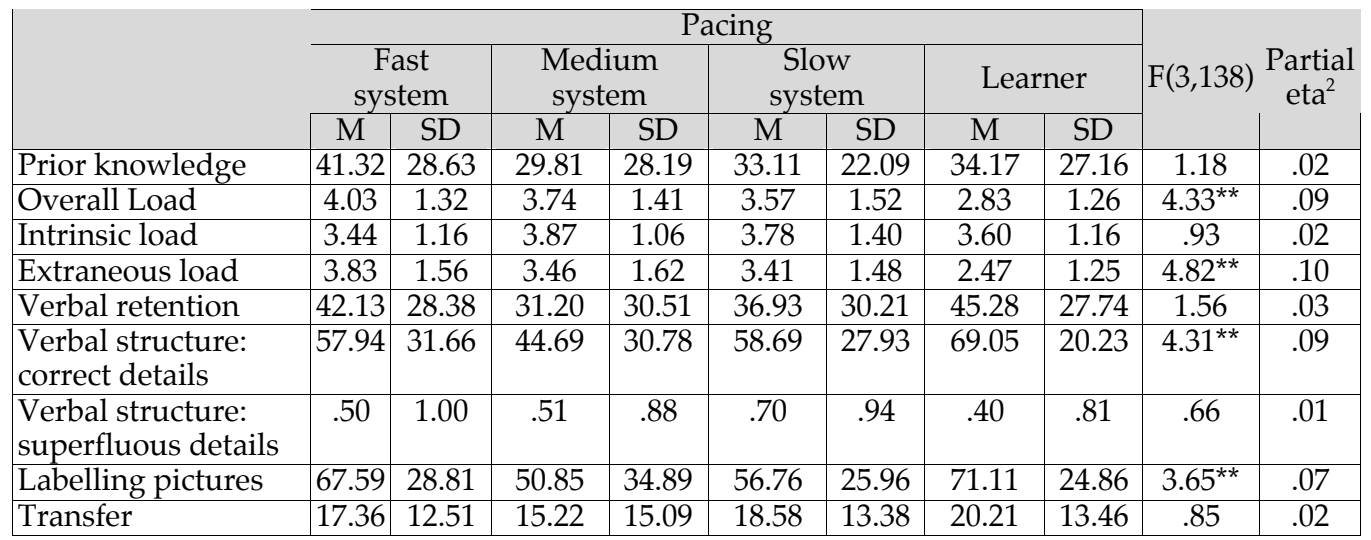

Pacing had no significant effects on verbal retention, reporting superfluous details in the verbal structure task, and transfer (see Table 4). For structural knowledge as measured by listing correct elements, a significant effect was found. With learner pacing, students reported more correct items in correct order than with system pacing (see Table 5). The system-paced groups also differed. Thereby, the medium system- 
paced group showed worse performance than the fast and slow system-paced groups, which did not differ significantly.

A significant effect of pacing occurred when students labelled pictures (see Table 4). With learner pacing, students could label more pictorial elements than with system pacing (see Table 5). Furthermore, the medium system-paced group showed worse performance than the fast system-paced group and did not significantly differ from the slow system-paced group, while the fast and the slow system-paced groups also did not differ significantly.

Table 5: Contrasts $\left({ }^{*} \mathrm{p} \leq .05 ;{ }^{* *} \mathrm{p} \leq .01 ;{ }^{* * *} \mathrm{p} \leq .001\right)$. Per each set of four one-sided tests: cumulative Alpha error controlled according to the Bonferroni-Holm correction (Holm, 1979)

\begin{tabular}{|l|c|c|c|c|}
\hline & Learner - system & Fast - medium & Fast - slow & Slow - medium \\
\hline Overall load & $\mathrm{t}(138)=-3.32^{* * *}$ & $\mathrm{t}(138)=-.89$ & $\mathrm{t}(138)=-1.42$ & $\mathrm{t}(138)=-.55$ \\
\hline Extraneous load & $\mathrm{t}(138)=-3.58^{* * *}$ & $\mathrm{t}(138)=-1.08$ & $\mathrm{t}(138)=-1.22$ & $\mathrm{t}(138)=-.16$ \\
\hline $\begin{array}{l}\text { Verbal structure } \\
\text { correct details }\end{array}$ & $\mathrm{t}(67.40)=3.27^{* * *}$ & $\mathrm{t}(138)=2.02^{*}$ & $\mathrm{t}(138)=.11$ & $\mathrm{t}(138)=2.15^{*}$ \\
\hline Labelling pictures & $\mathrm{t}(53.83)=2.37^{* *}$ & $\mathrm{t}(72.14)=2.27^{* *}$ & $\mathrm{t}(138)=1.59$ & $\mathrm{t}(70.10)=.84$ \\
\hline
\end{tabular}

\section{Discussion}

As was expected for this experiment, learner pacing of presentations proved to be more effective than system pacing (Betrancourt, 2005; Mayer, 2005). Allowing learners to pace the instruction with visual texts reduced the overall and extraneous mental effort while learning, and increased success in tasks of labelling pictures and structural knowledge. Overall, it would have been very surprising if intrinsic load had been affected by pacing, as the complexity of the learning material did not vary. This result especially is consistent with and replicates results obained by Stiller et al. (2009). To explain the superiority of learner pacing, learners were assumed to choose a pace that matches their individual speed of comprehension. In a cognitive load view, learner pacing is suitable to optimally reduce but not to eliminate temporal and visual splitattention, as well as concurrently allow for a better regulation of information processing. In general, we assume that individually pacing an instruction compensates split-attention effects, and thus extraneous load while learning is diminished. Consequently, this should allow an improved information processing and lead to better test performance.

For system-paced presentations, a main cause of pacing effects was suggested to be visual or temporal split-attention effects, depending on the speed of presentation. We assumed that a fast and slow pacing mainly lead to visual and temporal split-attention effects, respectively, and that a medium presentation speed should reduce but not eliminate visual and temporal split-attention effects. Hence, learners who learn with the medium system-paced instruction should have more success in the performance tasks and experience less mental effort than learners studying the fast and slow system-paced presentations. This perspective must be revised, because results do not correspond with this argumentation and the results reported in literature (Tabbers, 2002; Schmidt-Weigand, 2006). Three important aspects are considered in the following paragraphs.

Firstly, one of the main ideas behind cognitive load theory is that a more efficient use of working memory resources is needed when there is a risk of overload (Sweller, 
2005). Consequently, the use of visual text in system-paced multimedia instructions will only be harmful for learning when cognitive load is high. If not, the learner can compensate the increase in memory load by spending more mental effort and obtaining the same learning outcomes as with a more adequate presentation. On this background, looking at overall and extraneous mental effort in this study, it seems that the speeds of the system-paced instructions were overall moderate, and therefore not very load demanding. Presentations were maximally regarded as being in the middle and lower range of difficulty. The performance of the fast system-paced group especially indicates that visual split-attention did not play the dominant role as suspected. The mental effort ratings show that this was the most load demanding presentation, but mean ratings were in the medium range and not significantly higher than the other group ratings. Therefore, learners appeared to compensate the suspected badly designed presentation by investing more effort. Noteworthy is the result that the fast system-paced group was descriptively the most successful systempaced group, even approaching the level of performance of the learner-paced group. Generally, this questions the relevance of visual split-attention effects for the fast system-paced as well as the other system-paced learning condition.

Secondly, the slow system-paced group was assumed to suffer from temporal splitattention, therefore, being outperformed by the learner-paced group (Stiller et al., 2009). The fact that the means of performance were lower than with learner pacing also bolsters this assumption. But an alternative argument might better explain the results. In contrast to the slow system-paced instruction, a more rapid presentation could be more challenging to the learners, in order to overcome presentational handicaps, than a too-slow presentation. In other words, learners might be more interested in investing more mental effort to overcome working memory limits of capacity than limits in time as indicated by mental effort ratings. We might assume that a reduced processing time forces learners to process information more effectively in motivational aspects (Canelos et al., 1989; Schnotz, Fries \& Horz, 2009), as long as there is no threat of cognitive overload.

Thirdly, the main contradictory aspect is that the medium system-paced instruction proved to be the worst in terms of performance. This result was not expected, because we assumed that a medium pace allows the learners to suffer less from visual splitattention effects with fast presentations, and it concurrently reduces temporal splitattention effects, which occur when learners have to wait too long for the next bit of information. Moreover, this group showed the worst performance scores of all learning groups. One possible reason for the result could be related to motivation. This presentation mode could arguably have been less challenging, hence, learners did not try as hard as in the fast and slow system-paced group.

Summarised according to a motivational view, learner pacing might optimally trigger motivation, leading to an increased probability of engaging in meaningful processing, and thus to better test performance (Schnotz et al., 2009). This argumentation can already be found in older literature about learning with interactive computer instructions (Keller, 1983; Milheim \& Martin, 1991; Steinberg, 1989). Nevertheless, this proposed effect might be accompanied by reducing visual and temporal split-attention effects. For system pacing, as long as the presentation does not essentially hinder learning, or exceed information processing capacity by extraneous load, effects might better be explained in motivational aspects, because cognitive aspects might not play that dominant a role (Schnotz et al., 2009). 


\section{Conclusions}

Some limitations of this experiment must be acknowledged. The content covered is relatively limited, scientific, and factual. Study time was short and performance measures were not of the problem-solving type. In addition, the count-down from total playing time to zero per information unit in the system-paced conditions might have created a kind of spatial split-attention effect. Consequently, learners might have been distracted and have suffered from an additional disadvantage in these conditions. With these caveats in mind, the learner-paced instruction using visual texts was superior to the system-paced ones. Nevertheless, there was a strange pattern of results concerning performance and mental effort within the system-paced groups, which indicates that arguments using visual and temporal split-attention effects to explain less successful performance with system-paced instructions must be revisited and analysed in more detail. In addition, the results of the system-paced groups point to the possibility that the presentational speeds were not adequately varied; particularly the fast speed is suspected to have not been adequately chosen to create a visual splitattention effect. Thus, we might expect a different pattern of results if the speed is increased or slowed down. Therefore, we recommend using eye-tracking as a method to capture cognitive processing, controlling the learners' reading speed to be able to judge more easily if a presentation produces visual split-attention effect or not, and addressing motivational aspects. Thus, it should be possible to clarify results and to improve theoretical explanations.

In technical aspects, we advise to implement learner pacing for multimedia instructions consisting of static pictures and visual texts (Betrancourt, 2005; Mayer, 2005). When system-paced presentations are used, presentation time is a necessary consideration. As shown, giving more study time is not always beneficial for learners and might be even detrimental for performance (Canelos et al., 1989; Stiller et al., 2009). To account for this, multimedia designers should be given some empirically based rule to be able to calculate presentation time from text length and text difficulty. This can only be a suboptimal solution as we know that the individual reading speed and hence the individual speed of comprehension varies significantly between learners (SchmidtWeigand, 2006).

\section{References}

Ayres, P. \& Sweller, J. (2005). The split-attention principle in multimedia learning. In R. E. Mayer (Ed), The Cambridge handbook of multimedia learning (pp. 135-146). New York, NY: Cambridge University Press.

Betrancourt, M. (2005). The animation and interactivity principles in multimedia learning. In R. E. Mayer (Ed), The Cambridge handbook of multimedia learning (pp. 287-296). New York, NY: Cambridge University Press.

Bortz, J. (2005). Statistik für Human- und Sozialwissenschaftler [Statistics for human and social sciences]. Heidelberg: Springer.

Canelos, J., Dwyer, F., Taylor, W., Belland, J. \& Baker, P. (1989). The effect of embedded learning strategies in microcomputer-based instruction. Journal of Experimental Education, 57(4), 301318. http:/ / www.jstor.org/stable/ 20151781

Ginns, P. (2005). Meta-analysis of the modality effect. Learning and Instruction, 15(4), 313-331. http: / / dx.doi.org/10.1016/j.learninstruc.2005.07.001 
Hasler, B. S., Kersten, B. \& Sweller, J. (2007). Learner control, cognitive load and instructional animation. Applied Cognitive Psychology, 21(6), 713-729. http:/ / dx.doi.org/ 10.1002/acp.1345

Holm, S. (1979). A simple sequentially rejective multiple test procedure. Scandinavian Journal of Statistics, 6(2), 65-70. http:/ / www.jstor.org/ stable/4615733

Keller, J. M. (1983). Motivational design of instruction. In C. M. Reigeluth (Ed), Instructional design theories and models: An overview of their current status (pp. 383-434). Hillsdale, NJ: Lawrence Erlbaum.

Lepper, M. R. (1985). Microcomputers in education: Motivational and social issues. American Psychologist, 40(1), 1-18. http: / / psycnet.apa.org/ doi / 10.1037/ 0003-066X.40.1.1

Lusk, D. L., Evans, A. D., Jeffrey, T. R., Palmer, K. R., Wikstrom, C. S. \& Doolittle, P. E. (2008). Multimedia learning and individual differences: Mediating the effects of working memory capacity with segmentation. British Journal of Educational Technology, 40(4), 636-651. http: / / dx.doi.org/10.1111/j.1467-8535.2008.00848.x

Mayer, R. E. (2005). Principles for managing essential processing in multimedia learning: Segmenting, pretraining, and modality principles. In R. E. Mayer (Ed), The Cambridge handbook of multimedia learning (pp. 169-182). New York, NY: Cambridge University Press.

Mayer, R. E. \& Chandler, P. (2001). When learning is just a click away: Does simple user interaction foster deeper understanding of multimedia messages? Journal of Educational Psychology, 93(2), 390-397.

http:/ / www.sciencedirect.com/ science/ article / pii / S0022066302000491

Milheim, D. W. \& Martin, B. L. (1991). Theoretical basis for the use of learner control: Three different perspectives. Journal of Computer-Based Instruction, 18(3), 99-105.

Moreno, R. (2007). Optimizing learning from animations by minimizing cognitive load: Cognitive and affective consequences of signaling and segmentation methods. Applied Cognitive Psychology, 21(6), 765-781. http:/ / dx.doi.org/10.1002/ acp.1348

Niemiec, R. P., Sikorski, C. \& Walberg, H. J. (1996). Learner-control effects: A review of reviews and a meta-analysis. Journal of Educational Computing Research, 15(2), 157-164. http: / / dx.doi.org/10.2190/JV1U-EQ5P-X2PB-PDBA

Reeves, T. (1993). Pseudoscience in computer-based instruction: The case of learner control research. Journal of Computer-Based Instruction, 20, 39-46.

Schmidt-Weigand, F. (2006). Dynamic visualizations in multimedia learning: The influence of verbal explanations on visual attention, cognitive load and learning outcome. [viewed 16 Nov 2010]. http: / / geb.uni-giessen.de/geb/volltexte/2006/2699/

Schnotz, W., Fries, S. \& Horz, H. (2009). Motivational aspects of cognitive load theory. In M. Wosnitza, S. A. Karabenick, A. Efklides \& P. Nenniger (Eds), Contemporary motivation research: From global to local perspectives (pp. 69-96). Göttingen \& New York: Hogrefe \& Huber.

Schwan, S. \& Riempp, R. (2004). The cognitive benefits of interactive videos: Learning to tie nautical knots. Learning and Instruction, 14(3), 293-305. http: / / dx.doi.org/10.1016/j.learninstruc.2004.06.005

Sims, R. (1997). Interactivity: A forgotten art? Computers in Human Behavior, 13(2), 157-180. http:/ / dx.doi.org/10.1016/S0747-5632(97)00004-6 
Sims, R. (2000). An interactive conundrum: Constructs of interactivity and learning theory. Australian Journal of Educational Technology, 16(1), 45-57.

http:// www.ascilite.org.au/ajet/ajet16/sims.html

Steinberg, E. R. (1989). Cognition and learner control: A literature review, 1977-1988. Journal of Computer-Based Instruction, 16(4), 117-121.

Stiller, K. D., Freitag, A., Zinnbauer, P. \& Freitag, C. (2009). How pacing of multimedia instructions can influence modality effects: A case of superiority of visual texts. Australasian Journal of Educational Technology, 25, 184-203.

http:/ / www.ascilite.org.au/ajet/ajet25/stiller.html

Stiller, K. D. \& Zinnbauer, P. (2011). Does segmentation of complex instructional videos in big steps foster learning? The segmentation principle applied to a classroom video. In S.-M. Barton, J. Hedberg \& K. Suzuki (Eds), Proceedings of the Global Learn Asia Pacific 2011 - Global Conference on Learning and Technology (pp. 2044-2053). Chesapeake, VA: AACE. http: / / www.editlib.org/p/37442

Sweller, J. (2005). Implications of cognitive load theory for multimedia learning. In R. E. Mayer (Ed), The Cambridge handbook of multimedia learning (pp. 19-30). New York, NY: Cambridge University Press.

Tabbers, H. K. (2002). The modality of text in multimedia instructions. Refining the design guidelines. Heerlen: Open University of the Netherlands. [viewed 16 Nov 2010]. http: / / citeseerx.ist.psu.edu/viewdoc/ download?doi=10.1.1.99.6258\&rep=rep1\&type=pdf

Van Merriënboer, J. J. G. \& Kester, L. (2005). The four-component instructional design model: Multimedia principles in environments for complex learning. In R. E. Mayer (Ed), The Cambridge handbook of multimedia learning (pp. 71-93). New York, NY: Cambridge University Press.

Williams, M. D. (1996). Learner-control and instructional techniques. In D. H. Jonassen (Ed), Handbook of research for educational communications and technology (pp. 957-983). New York, NY: Macmillan.

Authors: Klaus D. Stiller, Kirstin Petzold and Peter Zinnbauer Department for Experimental Psychology, University of Regensburg 93040 Regensburg, Germany Author for correspondence: Klaus D. Stiller.

Email: Klaus.Stiller@psychologie.uni-regensburg.de Web: http: / / www-lukesch.uni-regensburg.de/Stiller/

Please cite as: Stiller, K. D., Petzold, K. \& Zinnbauer, P. (2011). Presentation time concerning system-paced multimedia instructions and the superiority of learner pacing. Australasian Journal of Educational Technology, 27(4), 693-708.

http: / / www.ascilite.org.au/ajet/ajet27/stiller.html 